\title{
Sensor Network Connectivity with Multiple Directional Antennae of a Given Angular Sum
}

\author{
Binay Bhattacharya Yuzhuang $\mathrm{Hu}$ \\ Qiaosheng Shi \\ School of Computing Science, Simon Fraser University \\ 8888 University Drive, Burnaby, BC V5A1S6, Canada \\ \{binay, yhu1, qshi1\}@cs.sfu.ca \\ Evangelos Kranakis \\ School of Computer Science, Carleton University \\ 1125 Colonel By Drive, Ottawa, Ontario K1S 5B6, Canada \\ kranakis@scs.carleton.ca \\ Danny Krizanc \\ Department of Mathematics and Computer Science, Wesleyan University \\ Middletown CT 06459, USA. \\ dkrizanc@wesleyan.edu
}

\begin{abstract}
We investigate the problem of converting sets of sensors into strongly connected networks of sensors using multiple directional antennae. Consider a set $S$ of $n$ points in the plane modeling sensors of an ad hoc network. Each sensor uses a fixed number, say $1 \leq k \leq 5$, of directional antennae modeled as a circular sector with a given spread (or angle) and range (or radius). We give algorithms for orienting the antennae at each sensor so that the resulting directed graph induced by the directed antennae on the nodes is strongly connected. We also study trade-offs between the total angle spread and range for maintaining connectivity.
\end{abstract}

\section{Introduction}

Sensors in use today can have a variety of antennae enabling them to vary their transmission range and orientation. A directional (or beam) antenna radiates greater power towards one or more directions thus allowing for increased performance on transmit and receive as well as reduced interference from unwanted sources. For example, ESPAR (Electronically Steerable Passive Array Radiator) antennae consisting of a steerable central source radiating within a region that can be approximated by a circular sector have been proposed by the engineering community. [13] and [16] study configuration and design of such multiple element antennae and present simulation results on their beam/null steering capability; they also found that 7-element ESPAR antennae can achieve continuous steering in almost all directions.

Directional antennae are known to enhance ad 
hoc network capacity and performance. The theoretical model presented in [7] shows that when $n$ omnidirectional antennae are optimally placed and assigned optimally chosen traffic patterns the transport capacity is $\Theta(\sqrt{W / n})$, where each antenna can transmit $W$ bits per second over the common channel(s). When both transmission and reception is directional [19] proves an $\sqrt{2 \pi / \alpha \beta}$ capacity gain as well as corresponding throughput improvement factors, where $\alpha$ is the transmission angle and $\beta$ is a parameter indicating that $\beta / 2 \pi$ is the average proportion of the number of receivers inside the transmission zone that will get interfered with. Additional experimental studies confirm the importance of using directional antennae in ad hoc networking. For example, [15] provides experimental evidence of throughput increase in ad hoc networks with beam forming antennas, [18] and [17] investigate bounds (depending on the specific antenna type and its parameters) on the amount of capacity gain that can be achieved when directional antennae perform independent communications in parallel, while [1] looks at transmission scheduling in ad hoc networks with directional antennae. See also [5] for MAC layer protocols that exploit the use of directional antennae.

\subsection{Antennae spreads and strong connectivity}

In this paper we are interested in studying connectivity properties of the underlying communication graph of a set of sensors using directional antennae. Unlike omnidirectional antennae, directional antennae give rise to a more complex directed graph whose connectivity varies depending on the direction of the beam. A directed edge $(u, v)$ exists between sensors $u$ and $v$ if and only if $v$ lies within the spread and range of $u$. It is important to maintain strong connectivity using the minimum possible antenna spread and range. Assuming each beam forming sensor has a given number of steerable directional antennae we are interested in providing an algorithm that minimizes the total antenna spread required so that by an appropriate rotation of the antennae the resulting network be- comes strongly connected. Thus, in the problems studied in the sequel, we assume that the antenna spread is part of the input.

Consider a set $S$ of $n$ points in the plane modeling sensors of an ad hoc network. Each sensor uses a directional antenna modeled by a circular sector with a given spread (or angle) and reach (or radius). A bound is given on the the spread of the antennae. Given a bound on the reach, the connectivity problem is to decide whether or not it is possible to orient the antennae so that the resulting directed graph induced by the directed antennae on the nodes is strongly connected. A variant of this problem concerns sensors with multiple antennae each, i.e., each sensor has $k$ antennae, for some given integer $k$. For a given bound on the sum of the angles of the antennae, it is of interest to know if there is a way to direct antennae with a given bound on their radius so that the resulting graph is strongly connected. Surprisingly, it will be shown that strong connectivity can still be maintained by appropriately rotating the antennae, even though the sum of angles of the directional antennae at each sensor is significantly less than the full angular spread $2 \pi$ of an omnidirectional antenna. We are interested in showing trade-offs between the number $k$ of antennae being used per sensor, the total spread of the antennae, and the maximum radius required per antennae, such that for suitable rotations of the antennae the network becomes strongly connected.

\subsection{Notation}

Let $S$ be a set of $n$ points in the plane. As usual, $d(x, y)$ is the Euclidean distance between two points $x$ and $y$ in the plane. Let $\varphi_{k}$ be a given non-negative value in $[0,2 \pi)$ such that the sum of angles of $k$ antennae at each sensor location is bounded by $\varphi_{k}$. Denote by $r_{k, \varphi_{k}}$ the minimum radius (or range) of directional antennae for a given $k$ and $\varphi_{k}$ that achieves strong connectivity under some rotation of the antennae. $T$ is a MST of $S$ with max degree $\leq 5, l_{\max }$ is the maximum length of edges in $T$. Note that $l_{\max }$ is a lower bound on $r_{k, \varphi_{k}}$ for any $k$ and $\varphi_{k}$. Without loss 
of generality, we assume that $l_{\max }=1$. This will be useful in the simplifying notation throughout the paper without compromising generality. Let $\delta(v)$ be the degree of vertex $v \in S$ in $T$. A degreeone vertex is arbitrarily chosen to be the root vertex of $T$, denoted by $R_{T}$. That is, $\delta\left(R_{T}\right)=1$. For each $v, v \in S$, let $T_{v}$ denote the subtree rooted at vertex $v$ and $v(1), v(2), \ldots, v(\delta(v)-1)$ denote its children sorted in counterclockwise order. For each $v, v \in S \backslash\left\{R_{T}\right\}$, let $p(v)$ be the parent of vertex $v$. Finally, $\widehat{u v w}$ denotes the counterclockwise angle between rays $\overrightarrow{v u}$ and $v \vec{w}$.

\subsection{Related work}

The problem of converting (connected) networks of omnidirectional sensors to strongly connected networks of sensors with directional antennae was first addressed in [4] where it was assumed that sensors had only one antenna each. They present a polynomial time algorithm achieving the optimal radius for the case when the sector angle of the antennae is at least $8 \pi / 5$. For smaller angles $\phi$ with $\pi \leq \phi<8 \pi / 5$ they give a polynomial time algorithm that computes an orientation of sectors of angle $\phi$ and radius $2 \sin (\pi-\phi / 2)$ so that the transmission graph is strongly connected. When the sector angle is smaller than $2 \pi / 3$, they show that the problem of determining the minimum radius in order to achieve connectivity is NP-hard. When antennae have spread 0 this problem turns out to be the bottleneck traveling salesman problem studied in [14]. It is shown in [14] that there is an algorithm which for any set of points in the plane computes antennae orientations such that the sensors have range $\leq 2$. Since $l_{\max }=1$ is a lower bound on the radius, the algorithms with radius greater than 1 above may thought of as approximation algorithms for the minimum radius required to achieve connectivity.

Other related papers include [9] on minimum range for energy consumption, [2] on energyefficient broadcasting in wireless networks, [3] on energy efficient wireless network design and [6] on the complexity of computing minimum energy consumption broadcast subgraphs. Topology con- trol for ad hoc networks with directional antennae is studied in [8], while directional versus omnidirectional antennae for energy consumption and $k$ connectivity of networks of sensors is considered in [11]. Coverage and connectivity in networks with directional sensors is investigated in [10]. Power consumption and throughput in mobile ad hoc networks using directional antennas is investigated in [12] while the performance of ad hoc networks with beamforming antennas is studied in [15]. However, to the best of our knowledge the problem of using directional antennae to achieve connectivity has not appeared in the literature before for the case of multiple antennae per sensor.

\subsection{Outline and results of the paper}

In Section 2 we provide necessary and sufficient conditions on antennae spreads so as to form a strongly connected graph with range equal to $l_{\max }=1$. These results provide optimal algorithms for large spread sum. In Section 3 we provide algo-

\begin{tabular}{|l|l|l|l|}
\hline$\#$ & Antennae Spreads & Antennae Range & Reference \\
\hline 1 & $\varphi_{1} \geq 0$ & 2 & {$[14]$} \\
& $\pi \leq \varphi_{1}<8 \pi / 5$ & $2 \sin \left(\pi-\frac{\varphi_{1}}{2}\right)$ & {$[4]$} \\
& $\varphi_{1} \geq 8 \pi / 5$ & 1 & {$[4]$} \\
\hline 2 & $\varphi_{2} \geq 0$ & 2 & {$[14]$} \\
& $\frac{2 \pi}{3} \leq \varphi_{2}<\pi$ & $2 \sin \left(\frac{\pi}{2}-\frac{\varphi_{2}}{4}\right)$ & Theorem 3 \\
& $\varphi_{2} \geq \pi$ & $2 \sin (2 \pi / 9)$ & Theorem 3 \\
& $\varphi_{2} \geq 6 \pi / 5$ & 1 & Theorem 2 \\
\hline 3 & $\varphi_{3} \geq 0$ & $\sqrt{3}$ & Theorem 5 \\
& $\varphi_{3} \geq 4 \pi / 5$ & 1 & Theorem 2 \\
\hline 4 & $\varphi_{4} \geq 0$ & $\sqrt{2}$ & Theorem 6 \\
& $\varphi_{4} \geq 2 \pi / 5$ & 1 & Theorem 2 \\
\hline 5 & $\varphi_{5} \geq 0$ & 1 & Folklore \\
\hline
\end{tabular}

Table 1. Upper bounds on antenna range for various specified sums of antennae.

rithms for two antennae of a given sum and prove the main result for two antennae per sensor. In Section 4 we discuss the case of more than two antennae. Table 1 summarizes the results obtained and 
also mentions previous results from the literature.

\section{Optimal Antennae Spreads and Connectivity}

For the given set of points, we will be referring to an arbitrary minimum weight spanning tree (MST) induced when edges between any two points are weighted by their corresponding Euclidean distance. Well-known geometric considerations imply that an MST of maximum degree 5 can be shown to exist. The first useful result we prove relates the node degree with the sum of the spreads of all the antennae.

Lemma 1 (Node degree and sum of antennae spreads) Assume that a node $u$ has degree $d$ and the sensor at $u$ is equipped with $k$ antennae, where $1 \leq k \leq d$, of range at least the maximum edge length of an edge from $u$ to its neighbors. Then $2(d-k) \pi / d$ is always sufficient and sometimes necessary bound on the sum of the angles of the antennae at $u$ so that there is an edge from $u$ to all its neighbors in an MST.

Proof. The result is trivially true for $k=d$ since we can satisfy the claim by directing a separate antenna to each node adjacent to $u$. So we can assume that $k \leq d-1$. To prove the necessity of the claim take a point at the center of a circle and with $d$ adjacent neighbors forming a regular $d$-gon on the perimeter of the circle of radius equal to the maximum edge length of the given spanning tree on $S$. Thus each angle formed between two consecutive neighbors on the circle is exactly $2 \pi / d$. It is easy to see that for this configuration a sum of $2(d-k) \pi / d$ is always necessary.

To prove that sum $2(d-k) \pi / d$ is always sufficient we argue as follows. Consider the point $u$ which has $d$ neighbors and consider the sum $\sigma$ of the largest $k$ angles formed by $k+1$ consecutive points of the regular polygon on the perimeter of the circle. We claim that $\sigma \geq 2 k \pi / d$. Indeed, let the $d$ consecutive angles be $\alpha_{0}, \alpha_{1}, \ldots, \alpha_{d-1}$. (see Figure 1). Consider the $d$ sums $\alpha_{i}+\alpha_{i+1}+\cdots+$ $\alpha_{i+k-1}$, for $i=0, \ldots, d-1$, where addition on the

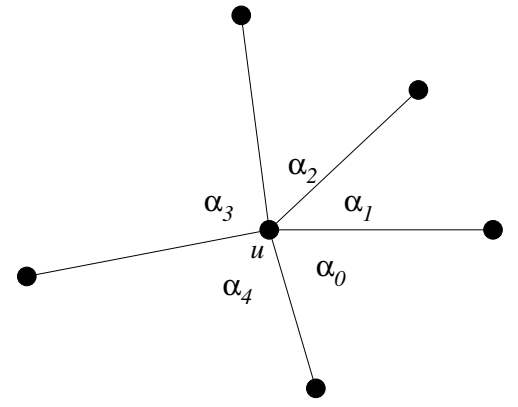

Figure 1. Example vertex with $d=5$.

indices is modulo $d$. Observe, that

$$
2 k \pi=\sum_{i=0}^{d-1}\left(\alpha_{i}+\alpha_{i+1}+\cdots+\alpha_{i+k-1}\right) \leq d \sigma .
$$

It follows that the remaining angles sum to at most $2 \pi-\sigma \leq 2 \pi-2 k \pi / d=2 \pi(d-k) / d$. Now consider the $k+1$ consecutive points, say $p_{1}, p_{2}, \ldots, p_{k+1}$, such that the sum $\sigma$ of the $k$ consecutive angles formed is at least $2 k \pi / d$. Use $k-1$ antennae each of size 0 radians to cover each of the points $p_{2}, \ldots, p_{k}$, respectively, and an angle of size $2 \pi(d-k) / d$ to cover the remaining $n-k+1$ points. This proves the lemma.

The next result we prove shows how antennae spreads affect the range in order to accomplish strong connectivity.

Theorem 2 For any $1 \leq k \leq 5$, if $\varphi_{k} \geq \frac{2(5-k) \pi}{5}$ then $r_{k, \varphi_{k}}=1$.

Proof. We prove the theorem by that if $\phi_{k} \geq 2(5-$ $k) \pi / 5$ then the antennae can be oriented in a way such that for every vertex $u$ there is a directed edge from $u$ to all its neighbors. Let $d$ be the degree of a vertex $u$ in an MST with max degree 5. If $d \leq k$ then for sure we have enough antennae.

Otherwise, $k<d \leq 5$. Then, $\phi_{k} \geq 2(5-$ $k) \pi / 5 \geq 2(d-k) \pi / d$. We know from Lemma 1 that for $k$ antennae $2(d-k) \pi / d$ is always sufficient and sometimes necessary on the sum of the angles of the antennae at $u$ so that there is a directional antenna from $u$ pointing to all its neighbors. Therefore, if $\phi_{k} \geq 2(5-k) \pi / 5$ then we can orient 
the antennae such that for every vertex $u$ there is a directed edge from $u$ to all its neighbors.

\section{Two Antennae}

In this section we prove the main theorem which provides a more detailed tradeoff between sum of antennae spreads and antennae range for the case of two antennae.

Theorem 3 Consider a set of $n$ sensors in the plane with two antennae each. There is an algorithm for directing the antennae so that the resulting graph is strongly connected such that the sum of angles and range of the antennae are as follows:

1. if $\varphi_{2}=\pi$ then $r_{2, \pi} \leq 2 \sin \left(\frac{2 \pi}{9}\right)$, and

2. if $\frac{2 \pi}{3} \leq \varphi_{2}<\pi$ then $r_{2, \varphi_{2}} \leq 2 \sin \left(\frac{\pi}{2}-\frac{\varphi_{2}}{4}\right)$.

Proof. (Theorem 3) We begin the proof by stating two simple facts.

Fact 1 (Refer to Figure 2(a).) For any vertices $u, v, w \in S$ such that $u$ and $w$ are adjacent neighbors of $v$ in $T$,

1. the angle $\widehat{u v w}$ is at least $\pi / 3$,

2. $d(u, w) \leq 2 \sin \left(\frac{\widehat{u v w}}{2}\right)$, and

3. the triangle $\triangle u v w$ is empty.

From Fact 1, we can easily derive the following fact.

Fact 2 (Refer to Figure 2(b).) Let $v_{1}, v_{2}, v_{3}, v_{4}, v_{5}$ be neighbors of a vertex $v \in S$ of degree 5 in $T$, sorted in counterclockwise order. Then,

1. the angles $\widehat{v_{1} v v_{2}}, \widehat{v_{2} v v_{3}}, \ldots, \widehat{v_{5} v v_{1}}$ are in $\left[\frac{\pi}{3}, \frac{2 \pi}{3}\right]$.

2. the angles $\widehat{v_{1} v v_{3}}, \widehat{v_{2} v v_{4}}, \ldots, \widehat{v_{5} v v_{2}}$ are in $\left[\frac{2 \pi}{3}, \pi\right]$.

Next we prove the results stated in the theorem by induction. In all cases (i.e., combination of $k$ and $\left.\varphi_{k}\right)$ we say that a rooted subtree $T_{v}(v \in S)$ satisfies Property 1 if for any imaginary point $p$ with $d(v, p) \leq r_{k, \varphi_{k}}$ (we use an imaginary point to

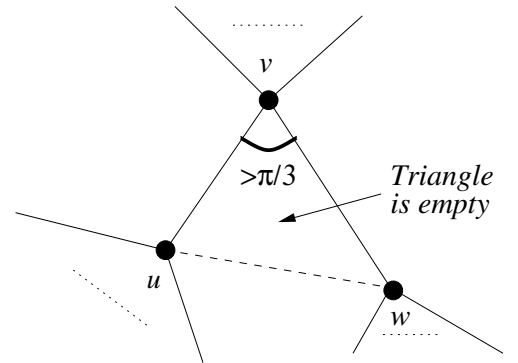

(a)

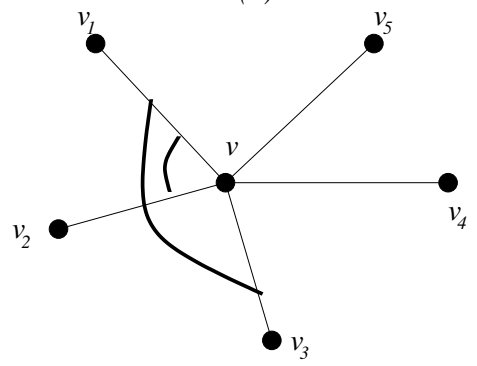

(b)

Figure 2. Examples for Facts 1 and 2.

simulate the parent or a sibling vertex of $v$ ), there exists a way to direct antennae located at vertices in $T_{v}$ such that the resulting graph is strongly connected and $p$ is covered by an antenna located at $v$. We have the following lemma.

Lemma 4 For each case in Theorem 3, if the rooted tree $T$ (rooted at $R_{T}$ ) satisfies Property 1 then there exists a way to direct antennae, where each antenna has radius $r_{k, \varphi_{k}}$ and each sensor has $k$ antennae whose angle sum is bounded by $\varphi_{k}$, such that the resulting graph is strongly connected.

Proof of Part 1: if $\varphi_{2}=\pi$ then $r_{2, \varphi_{2}} \leq$ $2 \sin \left(\frac{2 \pi}{9}\right)$

In this part of the proof, let us define $R_{2, \varphi_{2}}:=$ $2 \sin \left(\frac{2 \pi}{9}\right)$. Trivially, for any leaf $v \in S, T_{v}$ has Property 1 since we can use one antenna (located at $v$ ) of angle 0 and radius $R_{2, \varphi_{2}}$ to cover any imaginary point $p$ with $d(v, p) \leq R_{2, \varphi_{2}}$. For an internal vertex $u$, we assume that all rooted subtrees $T_{u(1)}, \ldots, T_{u(\delta(u)-1)}$ have Property 1. Next, we prove that $T_{u}$ has Property 1. Based on the degree 
of $u$ in $T$, we consider the following cases.

Case $\delta(u)=1,2:$ In this case, $u$ has one child $u(1)$. In fact, if $\delta(u)=1$ then $u=R_{T}$. According to assumption, $T_{u(1)}$ has Property 1 . Therefore, there is a way to direct antennae in $T_{u(1)}$ such that the resulting graph of vertices in $T_{u(1)}$ is strongly connected and $u$ is covered by an antenna located at $u(1)$. Refer to Figure 3(a). We then use two antennae (located at $u$ ) of angle 0 and radius $R_{2, \varphi_{2}}$. One antenna covers a given imaginary point $p$ with $d(u, p) \leq R_{2, \varphi_{2}}$ and the other one covers $u(1)$. In this way, the resulting graph of vertices in $T_{u}$ is strongly connected and $p$ is covered by an antenna located at $u$.

In the following, without loss of any generality, we assume that $u(1)$ is the first neighbor of $u$ when rotating the ray $\overrightarrow{u p}$.
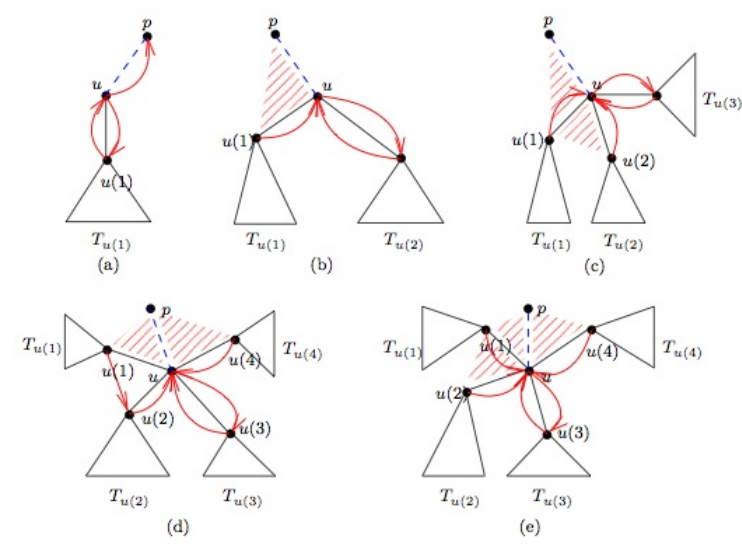

Figure 3. Case $\varphi_{2}=\pi$.

Case $\delta(u)=3$ : According to assumption, there is a way to direct antennae in $T_{u(1)}\left(\operatorname{resp} . T_{u(2)}\right)$ such that the resulting graph of vertices in $T_{u(1)}$ (resp. $\left.T_{u(2)}\right)$ is strongly connected and $u$ is covered by an antenna located at $u(1)$ (resp. $u(2))$. We can see that $\min \{\widehat{p u u(1)}, u(\widehat{1) u u(2)}, \widehat{u(2) u p}\} \leq$ $\frac{2 \pi}{3}\left(<\varphi_{2}\right)$. Suppose that $\widehat{p u u(1)} \leq \frac{2 \pi}{3}$. Refer to Figure 3(b). We then use two antennae (located at $u$ ) of radius $R_{2, \varphi_{2}}$. One antenna is of angle $\frac{2 \pi}{3}$ that covers the sector between rays $\overrightarrow{u p}$ and $u \overrightarrow{u(1)}$. The other one (of angle 0 ) covers $u(2)$. In this way, the resulting graph of vertices in $T$ is strongly connected and $p$ is covered by an antenna located at $u$.

Case $\delta(u)=4:$ It is easy to see that either $\widehat{p u u(2)}$ or $\widehat{u(2) u p}$ is no more than $\pi\left(=\varphi_{2}\right)$. Suppose $\widehat{p u u(2)} \leq \pi$. Refer to Figure 3(c). We then use two antennae (located at $u$ ) of radius $R_{2, \varphi_{2}}$. One antenna is of angle $\pi$ that covers the sector between rays $\overrightarrow{u p}$ and $u \overrightarrow{u(2)}$. The other one (of angle 0 ) covers $u(3)$.

Case $\delta(u)=5:$ Recall that $p$ lies in the sector between rays $u \overrightarrow{u(4)}$ and $u \overrightarrow{u(1)}$ (counterclockwise). We consider two cases depending on the location of $u$ 's predecessor.

If $p(u)$ lies in the sector between rays $u \overrightarrow{u(4)}$ and $u \overrightarrow{u(1)}$, then the angle $u\left(\widehat{4) u u(1)}\right.$ is in $\left[\frac{2 \pi}{3}, \pi\right]$ (by Fact 2). Accordingly,

$$
\min \left\{u(\widehat{1) u u(}), u(\widehat{2) u u(3)}, \overrightarrow{u(3) u(4)}\} \leq \frac{4 \pi}{9} .\right.
$$

Suppose that $u \widehat{(1) u u(2)} \leq \frac{4 \pi}{9}$. Refer to Figure 3(d). We then use two antennae (located at $u$ ) of radius $R_{2, \varphi_{2}}$. One antenna is of angle $\pi$ that covers the sector between rays $u \overrightarrow{u(4)}$ and $u \overrightarrow{u(1)}$. The other one (of angle 0) covers $u(3)$. According to assumption and $d(u(1), u(2)) \leq 2 \sin \left(\frac{2 \pi}{9}\right)(=$ $\left.R_{2, \varphi_{2}}\right)$, there is a way to direct antennae in $T_{u(1)}$ such that the resulting graph of vertices in $T_{u(1)}$ is strongly connected and $u(2)$ is covered by an antenna located at $u(1)$.

Otherwise, $p(u)$ does not lie in the sector between rays $u \overrightarrow{u(4)}$ and $u \overrightarrow{u(1)}$. Clearly, either the sector between rays $u \overrightarrow{u(1)}$ and $u \overrightarrow{u(2)}$ or the sector between rays $u \overrightarrow{u(3)}$ and $u \overrightarrow{u(4)}$ does not contain $p(u)$. Without loss of any generality, assume that the sector between rays $u \overrightarrow{u(1)}$ and $u \overrightarrow{u(2)}$ does not contain $p(u)$ (see Figure 3(e)). By Fact 2, the angle $u\left(\widehat{4) u u(2)}\right.$ is in $\left[\frac{2 \pi}{3}, \pi\right]$. We then use two antennae (located at $u$ ) of radius $R_{2, \varphi_{2}}$. One antenna is of 
angle $\pi$ that covers the sectors between rays $u \overrightarrow{u(4)}$

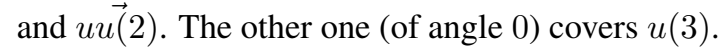

From the above discussions, we can see that $T_{u}$ has Property 1 . This completes the proof of the result for the case where $k=2$ and $\varphi_{2}=\pi$, that is, we are able to direct antennae in $T$ such that the resulting graph is strongly connected where radii of antennae is no more than $R_{2, \varphi_{2}}=2 \sin \left(\frac{2 \pi}{9}\right)$. This completes the proof of Part 1 of Theorem 3 in all cases.

Proof of Part 2: if $\frac{2 \pi}{3} \leq \varphi_{2}<\pi$ then $r_{2, \varphi_{2}} \leq 2 \sin \left(\frac{\pi}{2}-\frac{\varphi_{2}}{4}\right)$

In this part of the proof, let us define $R_{2, \varphi_{2}}:=$ $2 \sin \left(\frac{\pi}{2}-\frac{\varphi_{2}}{4}\right)$. The proof of the result for this case is similar to the one for the case $\varphi_{2}=\pi$. Here we only present the details for an internal vertex $u$ with $\delta(u) \geq 4$. Still, we assume that all rooted subtrees $T_{u(1)}, \ldots, T_{u(\delta(u)-1)}$ have Property 1 and that $u(1)$ is the first neighbor of $u$ when rotating the ray $\overrightarrow{u p}$.

Case $\delta(u)=4$ : There are three cases to consider depending on the size of the angle $u(\widehat{3) u u}(1)$.

If $u\left(\widehat{3) u u(1)} \leq \varphi_{2}\right.$, then we use two antennae (located at $u$ ) of radius $R_{2, \varphi_{2}}$. Refer to Figure 4(a). One antenna is of angle $\varphi_{2}$ that covers the sector between rays $u \overrightarrow{u(3)}$ and $u \overrightarrow{u(1)}$. The other one (of angle 0 ) covers $u(2)$.

Else if $u\left(\widehat{1) u u}(3) \leq \varphi_{2}\right.$, similar to the above. One antenna is of angle $\varphi_{2}$ that covers the sector between rays $u \overrightarrow{u(1)}$ and $u \overrightarrow{u(3)}$. The other one (of angle 0 ) covers $p$.

Otherwise, $u \widehat{(3) u u(1)}>\varphi_{2}$ and $u(\widehat{1) u u(3)}>$ $\varphi_{2}$. In this case, $\min \{\widehat{u(3) u p}, \widehat{p u u(1)}\}$ is at most $\frac{2 \pi}{3}$ which in turn is $\leq \varphi_{2}$. Suppose that $\widehat{u(3) u p} \leq$ $\varphi_{2}$. We then use two antennae (located at $u$ ) of radius $R_{2, \varphi_{2}}$. Refer to Figure 4(b). One antenna is of angle $\varphi_{2}$ that covers the sector between rays $u \overrightarrow{u(3)}$ and $\overrightarrow{u p}$. The other one (of angle 0) covers $u(1)$. We can see that $\min \{u(\widehat{1) u u(2)}, u(\widehat{2) u u(3)}\} \leq$ $\pi-\frac{\varphi_{2}}{2}$ since $u\left(\widehat{3) u u(1)}>\varphi_{2}\right.$. Therefore, either $u(1)$ or $u(3)$ is able to cover $u(2)$ with radius $\leq 2 \sin \left(\frac{\pi}{2}-\frac{\varphi_{2}}{4}\right)$.
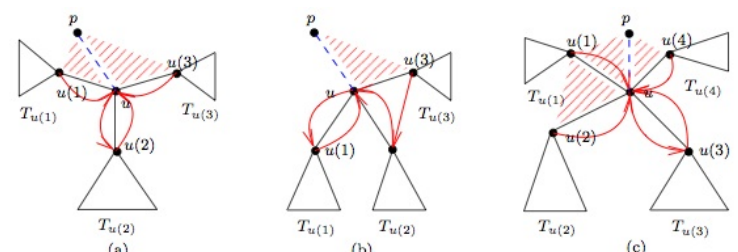

(a)

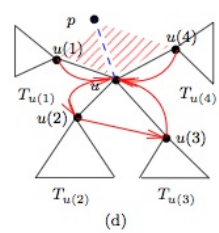

(b)
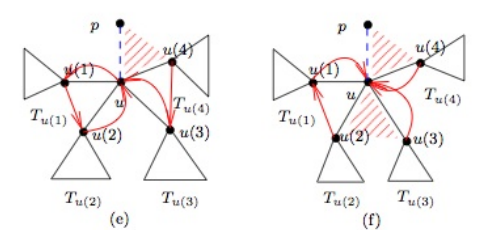

Figure 4. Case $\frac{2 \pi}{3} \leq \varphi_{2}<\pi$.

Case $\delta(u)=5:$ Recall that $p$ lies in the sector between rays $u \overrightarrow{u(4)}$ and $u \overrightarrow{u(1)}$ (counterclockwise). There are two cases to consider depending on the location of $u$ 's predecessor.

In the first case, if $p(u)$ does not lie in the sector between rays $u \overrightarrow{u(4)}$ and $u \overrightarrow{u(1)}$, then $u(\overrightarrow{4) u u(1)} \leq$ $\frac{2 \pi}{3} \leq \varphi_{2}$ (Fact 2).

- If the angle $u\left(\widehat{4) u u}(2) \leq \varphi_{2}\right.$ then one antenna is of angle $\varphi_{2}$ that covers the sector between rays $u \overrightarrow{u(4)}$ and $u \overrightarrow{u(2)}$. The other one (of angle 0 ) covers $u(3)$. (See Figure 4(c).)

- Otherwise, $u\left(\widehat{4) u u(}(2)>\varphi_{2}\right.$. It implies that $\min \left\{u(\widehat{1) u u(} 2), u(\widehat{2) u u(3)}\} \leq \pi-\frac{\varphi_{2}}{2}\right.$. Therefore, we can use one antenna (of angle $\left.\varphi_{2}\right)$ to cover the sector between rays $u \overrightarrow{u(4)}$ and $u \overrightarrow{u(1)}$ and the other one (of angle 0 ) to cover $u(2)$. Either $u(4)$ or $u(2)$ has one antenna to cover $u(3)$. (See Figure 4(d).)

In the second case, $p(u)$ also lies in the sector between rays $u \overrightarrow{u(4)}$ and $u \overrightarrow{u(1)}$ and there are two cases to consider.

1. If $\min \left\{\widehat{u(3) u p}, u(\widehat{4) u u(1)}, \widehat{p u u(2)}\} \leq \varphi_{2}\right.$, w.l.a.g., we assume that $\widehat{u(3) u p} \leq \varphi_{2}$. Then we can use one antenna located at $u$ (of angle $\varphi_{2}$ ) to cover the sector between rays $\overrightarrow{u \vec{u}(3)}$ and $\overrightarrow{u p}$ and the other one (of angle 0$)$ to cover $u(1)$. Either $u(3)$ or $u(1)$ 
has one antenna to cover $u(2)$ (note that $\min \{d(u(1), u(2)), d(u(3), u(2))\} \leq \sqrt{2}$ since $u(\widehat{1) u u(3)} \leq \pi) \cdot u(2)$ (resp. $u(4)$ ) has one antenna to cover $u$.

2. Otherwise, $\widehat{u(3) u p}, u(\widehat{4) u u(1)}, \widehat{p u u(2)}$ are larger than $\varphi_{2}$. We have $\widehat{u(3) u p} \leq \pi$ and $\widehat{\operatorname{puu}(2)} \leq \pi$ since $\varphi_{2} \geq \frac{2 \pi}{3}$ and $u \widehat{(2) u u(3)} \geq$ $\frac{\pi}{3}$. Without loss of generality we may assume that $\widehat{u(4) u p} \leq \widehat{p u u(1)}$. Then $\widehat{u(4) u p} \leq \varphi_{2}$ since $\varphi_{2} \geq \frac{2 \pi}{3}$.

(a) If $\widehat{u(4) u p} \geq \frac{\varphi_{2}}{2}$, then $\widehat{p u u(1)} \geq \frac{\varphi_{2}}{2}$. We can see that

$$
\begin{aligned}
& \widehat{u(3) u p} \leq \pi, \widehat{p u u(2)} \leq \pi \\
\Rightarrow \quad & u\left(\widehat{(1) u u(}(2) \leq \pi-\frac{\varphi_{2}}{2},\right. \\
& u\left(\widehat{(3) u u(}(4) \leq \pi-\frac{\varphi_{2}}{2}\right. \\
\Rightarrow \quad & d(u(1), u(2)) \leq 2 \sin \left(\frac{\pi}{2}-\frac{\varphi_{2}}{4}\right), \\
& d(u(4), u(3)) \leq 2 \sin \left(\frac{\pi}{2}-\frac{\varphi_{2}}{4}\right) .
\end{aligned}
$$

Therefore, we use one antenna (of angle $\varphi_{2}$ ) to cover the sector between rays $u \overrightarrow{u(4)}$ and $\overrightarrow{u p}$ and the other one (of angle $0)$ to cover $u(1)$. Also, $u(1)$ (resp. $u(4))$ has one antenna to cover $u(2)$ (resp. $u(3))$. See Figure 4(e).

(b) Otherwise, $\widehat{u(4) u p}<\frac{\varphi_{2}}{2}$. We can see that $u\left(\widehat{4) u u(1)} \geq \varphi_{2} \Rightarrow \widehat{p u u(1)}>\right.$ $\frac{\varphi_{2}}{2} \Rightarrow u\left(\widehat{1) u u(2)}<\pi-\frac{\varphi_{2}}{2} \Rightarrow\right.$ $d(u(1), u(2))<2 \sin \left(\frac{\pi}{2}-\frac{\varphi_{2}}{4}\right)$.

i. If $u \widehat{(2) u u(3)} \leq \frac{\varphi_{2}}{2}$ then we use one antenna (of angle $\frac{\varphi_{2}}{2}$ ) to cover the sector between rays $u \overrightarrow{u(4)}$ and $\overrightarrow{u p}$ and the other one (of angle $\frac{\varphi_{2}}{2}$ ) to cover the sector between rays $u \overrightarrow{u(2)}$ and $u \overrightarrow{u(3)}$. Also, $u(2)$ has one antenna to cover $u(1)$. See Figure 4(f).

ii. Otherwise, $u \widehat{(2) u u(3)}>\frac{\varphi_{2}}{2}$. Then, $u\left(\widehat{3) u u(4)}<\pi-\frac{\varphi_{2}}{2} \Rightarrow\right.$ $d(u(3), u(4))<2 \sin \left(\frac{\pi}{2}-\frac{\varphi_{2}}{4}\right)$. Therefore, we use one antenna (of angle $\varphi_{2}$ ) to cover the sector between rays $u \overrightarrow{u(4)}$ and $\overrightarrow{u p}$ and the other one (of angle 0 ) to cover $u(1)$. Also, $u(1)$ (resp. $u(4))$ has one antenna to cover $u(2)$ (resp. $u(3)$ ). See Figure 4(d).

This completes the proof of Part 2 of Theorem 3 in all cases and therefore the proof of the main theorem is now complete.

\section{More than two Antennae}

In this section we consider the case of more than two antennae. To begin with, observe that since every set of points in the plane has a Euclidean spanning tree of degree at most 5 , it is easy to see that for any set $S$ of points in the plane, with five antennae per sensor, we can strongly connect the sensors using a range of at most 1 . Therefore it remains to consider the cases of three and four antennae.

\section{Three antennae per sensor}

Theorem 5 For any set $S$ of points in the plane, with three antennae per sensor, we can strongly connect the sensors using a range of at most $\sqrt{3}$.

Proof. Assume we have three antennae with angles 0 at each sensor. The proof is by induction on the height of the tree. The inductive hypothesis that must be maintained throughout is the following: "Given a rooted directional tree we can assign antennae so that the resulting graph is strongly connected while the out degree of the root never exceeds 2." Assuming the inductive hypothesis is true we argue as follows. Take as root any vertex, say $u$, of an MST. Vertex $u$ has at most $d \leq 5$ subtrees, with corresponding roots $u_{1}, u_{2}, \ldots, u_{d}$, respectively. By the induction hypothesis we can assign antennae in the subtrees so that the resulting graphs are strongly connected and the vertices $u_{i}$ use at most two antennae each. It follows that we can direct the remaining antenna at $u_{i}$ towards the root $u$. 
It remains to show how to direct two antennae from $u$ to its $d$ children so that the range being used does not exceed $\sqrt{3}$. By the previous construction, if it is satisfied for all the subtrees of $u$ it must also be satisfied for the tree rooted at $u$. Therefore it remains to examine the initial cases of the induction hypothesis. To this end consider a tree consisting of

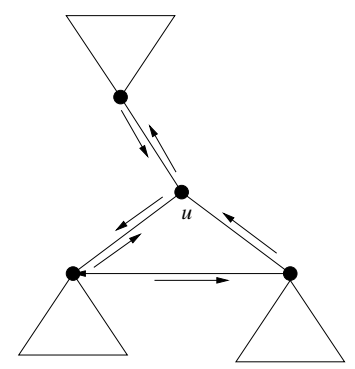

(a)

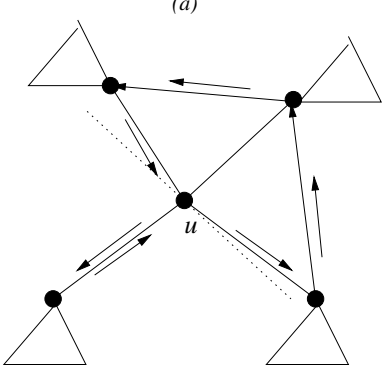

(b)

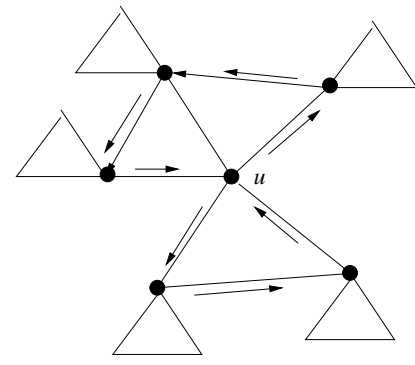

(c)

Figure 5. Directing antennae among $u$ and its children so as to guarantee that the maximum range does not exceed $\sqrt{3}$. Notice that in all three cases the out degree of $u$ is 2 .

the root $u$ and the $d$ vertices $u_{1}, \ldots, u_{d}$ adjacent to $u$. The result is trivial if $d \leq 2$. If $d=3$ then as de- picted in Figure 5(a) we find an angle between two children of $u$ forming an angle $\leq 2 \pi / 3$ and connect them with a directed edge. If $d=4$ then as depicted in Figure 5(b) we find two adjacent angles among three children of $u$ each forming an angle $\leq 2 \pi / 3$ and connect them with directed edges. Finally, if $d=5$ then as depicted in Figure 5(c) we find three angles among the children of $u$ each forming an angle $\leq 2 \pi / 3$ and connect them with directed edges. This completes the proof of Theorem 5 .

\section{Four antennae per sensor}

Theorem 6 For any set $S$ of points in the plane, with four antennae per sensor, we can strongly connect the sensors using a range of at most $\sqrt{2}$.

Proof. This is similar to the proof of Theorem 5. Assume we have four antennae with angles 0 at each sensor. The proof is by induction on the height of the tree. The inductive hypothesis that must be maintained throughout is the following: "Given a rooted directional tree we can assign antennae so that the resulting graph is strongly connected while the out degree of the root never exceeds 3." Assuming the inductive hypothesis is true we argue as follows. Take as root any vertex, say $u$, of an MST. Vertex $u$ has at most $d \leq 5$ subtrees, with corresponding roots $u_{1}, u_{2}, \ldots, u_{d}$, respectively. By the induction hypothesis we can assign antennae in the subtrees so that the resulting graphs are strongly connected and the vertices $u_{i}$ use at most three antennae each. It follows that we can direct the remaining antenna at $u_{i}$ towards the root $u$.

It remains to show how to direct two antennae from $u$ to its $d$ children so that the range being used does not exceed $\sqrt{2}$. By the previous construction, if it is satisfied for all the subtrees of $u$ it must also be satisfied for the tree rooted at $u$. Therefore it remains to examine the initial cases of the induction hypothesis. To this end consider a tree consisting of the root $u$ and the $d$ vertices $u_{1}, \ldots, u_{d}$ adjacent to $u$. The result is trivial if $d \leq 3$. If $d=4$ then as depicted in Figure 6(a) we find an angle between two children of $u$ forming an angle $\leq \pi / 2$ and connect them with a directed edge. Finally, if $d=5$ then as depicted in Figure 6(b) we find two angles among 

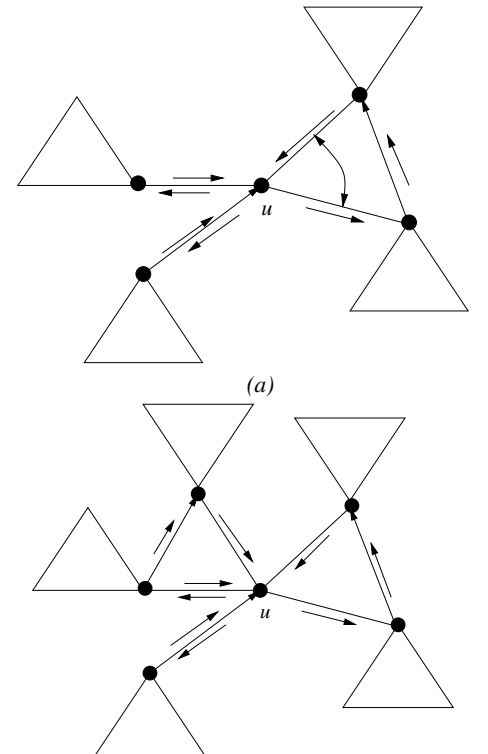

(b)

Figure 6. Directing antennae among $u$ and its children so as to guarantee that the maximum range does not exceed $\sqrt{2}$. Notice that in both cases the out degree of $u$ is 3 .

the children of $u$ each forming an angle $\leq \pi / 2$ and connect them with directed edges. This completes the proof of Theorem 6 .

\section{Conclusion}

We gave several tradeoffs between the total antenna spread and range when each sensor has $k$ antennae, $k=2,3,4$, so as to guarantee the resulting network is strongly connected. In addition to studying tradeoffs arising from results obtained in this paper several problems remain open. Lower bounds are lacking from our study and it remains open to prove NP completeness results for the case of multiple antennae per sensor. Another interesting question concerns ensuring that for a given integer $c$ the resulting network is strongly $c$-connected, i.e., it remains strongly connected after the deletion of any $c-1$ nodes.
In a real network, one has to consider interference from nearby links to be able to judge the connectivity of the network. In this study the system model assumes that there is no interference. It is a challenge work to obtain similar results that can be applied to the real world.

\section{References}

[1] L. Bao and JJ Garcia-Luna-Aceves. Transmission scheduling in ad hoc networks with directional antennas. Proceedings of the 8 th annual international conference on Mobile computing and networking, pages 48-58, 2002.

[2] I. Caragiannis, C. Kaklamanis, and P. Kanellopoulos. New Results for Energy-Efficient Broadcasting in Wireless Networks. Proc. of the 13th Annual International Symposium on Algorithms and Computation (ISAACO2), pages 332-343, 2002.

[3] I. Caragiannis, C. Kaklamanis, and P. Kanellopoulos. Energy-Efficient Wireless Network Design. Theory of Computing Systems, 39(5):593-617, 2006.

[4] I. Caragiannis, C. Kaklamanis, E. Kranakis, D. Krizanc, and A. Wiese. Communication in Wireless Networks with Directional Antennae. In proceedings of 20th ACM Symposium on Parallelism in Algorithms and Architectures (SPAA'08) Munich, Germany, June 14 - 16, pages 344-351, 2008.

[5] R.R. Choudhury, X. Yang, R. Ramanathan, and N.H. Vaidya. Using directional antennas for medium access control in ad hoc networks. Proceedings of the 8th annual international conference on Mobile computing and networking, pages 59-70, 2002.

[6] A.E.F. Clementi, P. Crescenzi, P. Penna, G. Rossi, and P. Vocca. On the complexity of computing minimum energy consumption broadcast subgraphs. Proceedings of the 18th 
Annual Symposium on Theoretical Aspects of Computer Science, 2010:121-131, 2001.

[7] P. Gupta and PR Kumar. The capacity of wireless networks. IEEE Transactions on Information Theory, 46(2):388-404, 2000.

[8] Z. Huang, C.C. Shen, C. Srisathapornphat, and C. Jaikaeo. Topology control for ad hoc networks with directional antennas. Proceedings. Eleventh International Conference on Computer Communications and Networks, pages 16-21, 2002.

[9] L.M. Kirousis, E. Kranakis, D. Krizanc, and A. Pelc. Power consumption in packet radio networks. Theoretical Computer Science, 243(1-2):289-305, 2000.

[10] E. Kranakis, D. Krizanc, and J. Urrutia. Coverage and Connectivity in Networks with Directional Sensors. Proceedings Euro-Par Conference, Pisa, Italy, August, pages 917924, 2004.

[11] E. Kranakis, D. Krizanc, and E. Williams. Directional versus omnidirectional antennas for energy consumption and k-connectivity of networks of sensors. Proceedings of OPODIS, 3544:357-368, 2004.

[12] A. Nasipuri, K. Li, and UR Sappidi. Power consumption and throughput in mobile ad hoc networks using directional antennas. Proceedings. Eleventh International Conference on Computer Communications and Networks, pages 620-626, 2002.

[13] T. Ohira and K. Gyoda. Electronically steerable passive array radiator antennas forlowcost analog adaptive beamforming. Proceedings of IEEE International Conference on Phased Array Systems and Technology, pages 101-104, 2000.

[14] R.G. Parker and R.L. Rardin. Guaranteed performance heuristics for the bottleneck traveling salesman problem. Oper. Res. Lett, 2(6):269-272, 1984.
[15] R. Ramanathan. On the performance of ad hoc networks with beamforming antennas. Proceedings of the 2nd ACM international symposium on Mobile ad hoc networking \& computing, pages 95-105, 2001.

[16] R. Schlub, J. Lu, and T. Ohira. Seven-element ground skirt monopole ESPAR antenna design from a genetic algorithm and the finite element method. Antennas and Propagation, IEEE Transactions on, 51(11):30333039, 2003.

[17] A. Spyropoulos and CS Raghavendra. Energy efficient communications in ad hoc networks using directional antennas. INFOCOM 2002. Twenty-First Annual Joint Conference of the IEEE Computer and Communications Societies. Proceedings. IEEE, 1:220-228, 2002.

[18] A. Spyropoulos and CS Raghavendra. Capacity bounds for ad-hoc networks using directional antennas. ICC'03, IEEE International Conference on Communications, 1:348-352, 2003.

[19] S. Yi, Y. Pei, and S. Kalyanaraman. On the capacity improvement of ad hoc wireless networks using directional antennas. Proceedings of the 4th ACM international symposium on Mobile ad hoc networking \& computing, pages 108-116, 2003. 